\title{
Effect of a Fuselage Boundary Layer Ingesting Propulsor on Airframe Forces and Moments
}

\author{
Antonio Martínez Fernández ${ }^{\text {a, * }}$, Howard Smith ${ }^{\text {a }}$ \\ a Cranfield University, College Road, Cranfield, Bedfordshire, MK43 OAL, UK
}

\begin{abstract}
The aim of this research project was to investigate the benefits of hybrid-distributed propulsion and boundary layer ingestion applied to an airliner similar in size, range and cruise velocity to an Airbus A320 or a Boeing 737-800. The power system selected consisted of two under-wing mounted conventional turbofans and an electrically driven boundary layer ingesting fan, located at the rear fuselage. The power required for the fan is extracted from both turbofans. The worked carried out and presented in this paper offers a new approach to modelling boundary layer ingestion configurations, consisting of using the sliding mesh method to simulate the rotation of the blades within the airflow. The simulations indicate that ingesting the boundary layer results in remarkable improvements reducing drag and increasing propulsive force, reaffirming the potential of this technology to reduce fuel consumption. However, the results also registered large changes in the pressure distribution, and hence in the pitching, rolling and yawing moment that need to be accounted for in the design phase.
\end{abstract}

Keywords: Aircraft Design, Boundary Layer Ingestion, Computational Fluid Dynamics

\section{Introduction}

Boundary layer ingestion has stood out as a solution to improve both aerodynamics and propulsion performance [1-3]. The aim of ingesting the boundary layer is to reenergize the slower air moving near the surface of the body to reduce the drag due to friction. This can be achieved by placing a propulsor at the rear of the body such that the boundary layer is accelerated passing through it.

The benefits of ingesting the boundary layer around a body are not limited to the reduction of drag but also to improve engine performance. The airflow at the intake of the engine in boundary layer ingesting concepts is slower than the freestream ingested by conventional configurations, where the engine is placed far from the body. Therefore, the ram drag is reduced in the former design.

However, the engines are designed to be embedded in the freestream, where the flow is continuous and uniform. On the other hand, the boundary layer at the rear of the fuselage is highly turbulent, which produces severe and uneven stresses in the fan blades and the nacelle that can reduce engine performance [4].

The potential of this configuration to reduce drag and fuel consumption has made it target of numerous aircraft designs such as the NASA's D8 and N3-X or the E-Thrust, concept developed by Airbus and Rolls-Royce. However, the level of sophistication of these models require to overcome technological and certification challenges that delay a realistic entry into service. On the other hand, aircraft such as the STARC-ABL, also conceived by NASA, have adopted more traditional approaches, incorporating an electric fan at the rear of the tail cone, ingesting the boundary layer around the fuselage in a conventional wingtube configuration.

Between 2010 and 2015, Boeing Research \& Technology conducted a project called SUGAR (Subsonic Ultra Green Aircraft Research) [5], issued by NASA, to explore Hybrid electric propulsion. The project, led by Marty K. Bradley and Cristopher K. Bradley included a configuration with a boundary layer ingesting aft-propulsor. The configuration also included two LNG (Liquid Natural Gas) fuel cell hybrid turbines to a truss braced wing. Due to the level of technology considered in this airplane the design was categorized as an $\mathrm{N}+4$ generation (entry into service 2040 2050). The results of the study indicated a saving of 10 percent in fuel consumption with respect to a similar configuration without boundary layer ingestion $[5,6]$.

One year later, based on the SUGAR project, Jason R. Welstead and James L. Felder, from the NASA Langley Research Center and the NASA Glenn Research Center respectively, presented their own BLI concept, the STARC$\mathrm{ABL}$ [7]. This design adopted a more traditional configuration and, excluding the aft-fan, the other elements of the aircraft remained similar to the ones found in today's airliners. The less sophisticated technology assumptions reduced the entry into service to 2035 . The NASA design

* Corresponding author.

Email: antoniomfernd@gmail.com (Antonio Martínez Fernández) 


\begin{tabular}{|llll|}
\hline & Mass flow rate & AR & \\
$\dot{m}$ & Reference area & BLI & Boundary layer ingestion \\
$\mathrm{S}_{\text {ref }}$ & Freestream velocity & CAD & Computer added design \\
$\mathrm{U}_{\infty}$ & drag & OEW & Operative empty weight \\
$\mathrm{D}$ & Force & CFD & Computational fluid dynamics \\
$\mathrm{F}$ & Thrust & HPC & High performance computer \\
$\mathrm{T}$ & Air velocity & MTOW & Max take-off weight \\
$\mathrm{u}$ & Air density & & \\
$\rho$ & & & \\
\hline
\end{tabular}

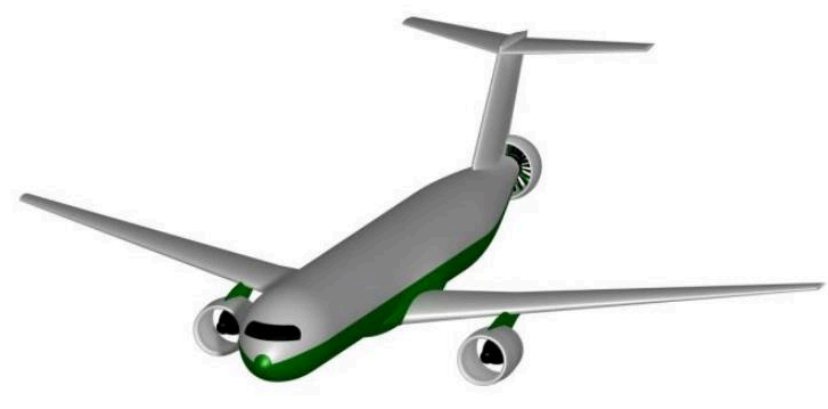

Fig 1. BLI concept

offers a potential $12 \%$ mission fuel burn reduction with respect to a conventional airliner with the same technology assumptions [7].

The significant potential of this configuration, to improve both aerodynamics and engine performance, motivated NASA to continue the research in boundary layer ingestion with more detailed analyses. In 2017, Justin S. Gray, from the NASA Glenn Research Center, expanded Welstead and Felder's work by developing a fully coupled propulsionRANS model applied to a boundary layer ingestion configuration [8]. Before Gray's research, the analyses in boundary layer ingestion considered the effects of the aerodynamics on the propulsor or the effects of the propulsion system on the aerodynamics but not both simultaneously [8]. Gray developed a reciprocal process using the RANS solver ADflow for the aerodynamics model and the 1D thermodynamic cycle tool pyCycle to model the propulsion system [8]. Gray concluded that a boundary layer ingestion configuration produces improvements in performance due to both propulsion and aerodynamics equally and considering only aerodynamics or propulsion ignores half of the overall effect [8]. The results obtained demonstrated the need to use a fully coupled method to account for both aerodynamics and propulsion system simultaneously to capture the real benefits of boundary layer ingestion.

Additionally, Mark J. Drela and Aurélien Arntz developed methodologies based on power balance [9] exergy-based formulation [10] respectively that highlight the importance of considering the full integration between the propulsor and the airframe. Furthermore, in 2018, research conducted

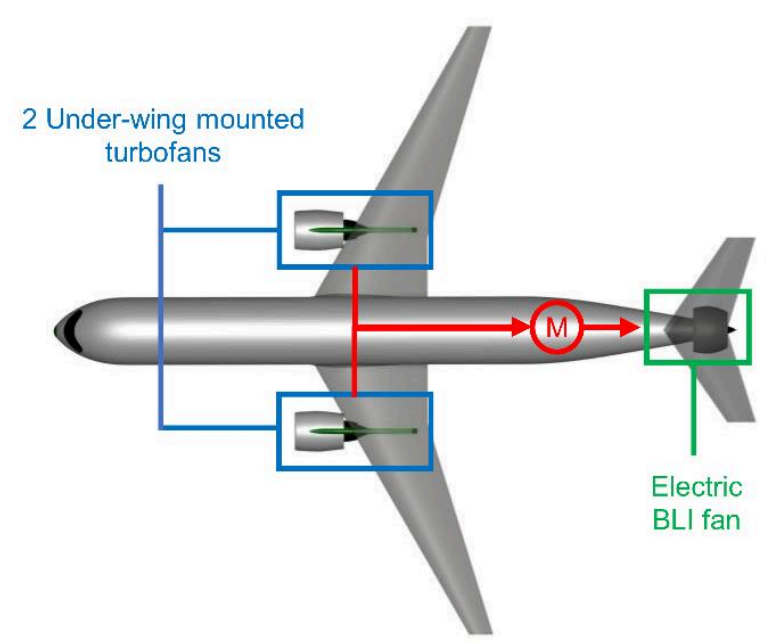

Fig 2. BLI power system setting

by Cranfield University and NASA Glenn Research Center by C. Goldberg, D. Nalianda, D. MacManus, P. Pilidis and J. Felder, included a methodology to study BLI performance for a range of flight conditions and propulsion system power settings at off-design conditions.

The research projects conducted on BLI focused on propulsive force improvements and aerodynamic enhancement in the direction of flight and it has been well documented in both fields. However, in the previous research on BLI, simplifications were done to model the configurations such as $2 \mathrm{D}$ representations or axisymmetric fuselages. Although these simplifications are valid for a preliminary study on the effects of boundary layer ingestion and examine the forces in the direction of flight, it does not capture the ramifications of ingesting the boundary layer in a real 3-dimmensional and non-axisymmetric geometry. Therefore, there is a niche in the current research on BLI assessment regarding how this technology affects the other components of the forces acting of the aircraft.

The aim of this project was to investigate the threedimensional changes, in forces and moments, while ingesting the boundary layer around the fuselage of a single-aisle airliner, with respect to a conventional configuration, through CFD simulation. The main advantage of using CFD is that it can be applied to an accurate 3D representation of the elements that compose the aircraft, without the need of adopting sever simplifications. This, is crucial to examine the different profiles of the boundary 


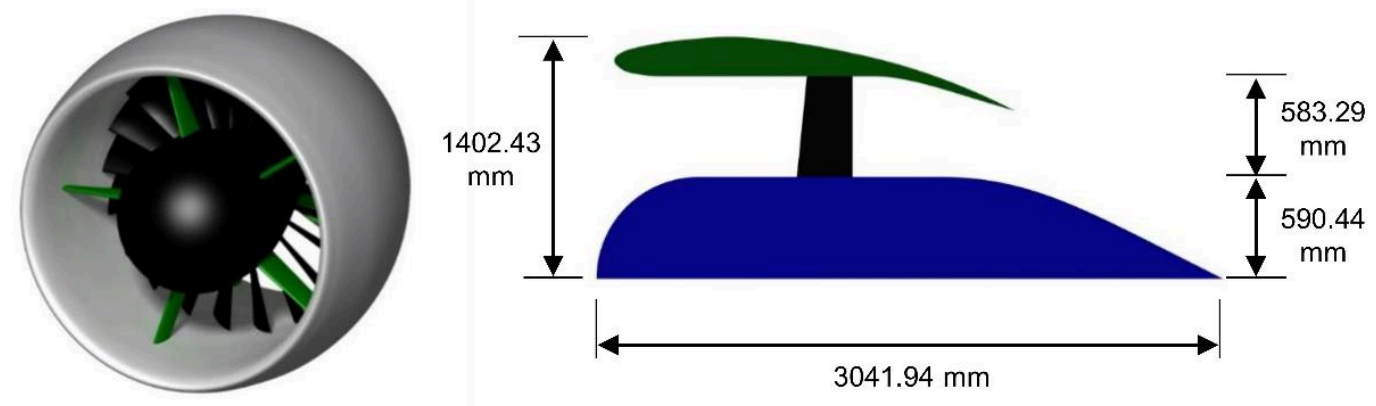

Fig 3. Electric fan CAD render (left) and cross-section (right)

\begin{tabular}{lcr}
\hline \multicolumn{2}{c}{ Table 1: BLI Configuration Features } \\
${ } }$ & Units & Value \\
\hline Range & $\mathrm{nm}$ & 3500 \\
Cruise Mach & - & 0.78 \\
Cruise Altitude & $\mathrm{m}$ & 11,79 \\
MTOW & $\mathrm{kg}$ & 73,99 \\
OEW & $\mathrm{kg}$ & 38,22 \\
Wing Area & $\mathrm{m}^{2}$ & 137.85 \\
Wing Span & $\mathrm{m}$ & 36 \\
AR & - & 12 \\
Leading Edge Sweep & Degrees & 30 \\
Max T-O Field length & $\mathrm{m}$ & 2500 \\
Thrust (cruise) & $\mathrm{kN}$ & 40.62 \\
\hline
\end{tabular}

layer depending not only in the longitudinal component of fuselage and propulsor but also at different radial locations. The goal is to analyze the three-dimensional distributions of pressure acting on the integrated elements to attain insight of the aerodynamic coefficients as well as the pitching, rolling and yawing moments.

\section{Concept Description and Design}

The BLI configuration selected for this research consisted of a narrow-body airliner designed to be similar in size, range and cruise velocity to an Airbus A320 or a Boeing 737-800 (Fig 1). The power system included two under-wing mounted turbofans and an electric fan attached to the tail cone, ingesting the boundary layer around the fuselage. The power required to drive the fan is extracted from two generators located in each turbofan (Fig 2). The main features of the concept are summarized in Table 1

\subsection{Aircraft Design}

The conceptual design of the aircraft was completed in two different phases. The first stage included the design of a conventional airliner configuration, which acted as a baseline to build upon during the development of the BLI concept in the second step of the design process. In addition, the baseline configuration was also used as a comparison point to evaluate the changes produced by the ingestion of the boundary layer.

The mission profile was based upon those of the Airbus A320 and Boeing 737-800 and the performance requirements were constructed through a market study and a parametric analysis taking into account take off, second segment climb, approach and cruse constrains $[11,12]$. The size of the wing for both configurations was determined from the wing loading and optimized for Mach cruise. A T-tail arrangement was selected for the empennage due to the minimum aerodynamic interference with the aft-fan that it provides. It was sized using the tail volume coefficient method [12]. The fuselage was intended to accommodate 160 passengers in a 2-class arrangement, 18 seats were allocated to first class and the remaining 142 were distributed in Economy Class.

\subsection{Electric Fan Design}

The electric fan, displayed in Fig 3, was outlined based upon the "Fuselage Fan" used by Boeing in the SUGAR project [5]. The convergent nacelle was sized following the same process taken by Welstead and Felder during the development of the STARC-ABL, based on the relation between the deficit of momentum captured and the percentage of the boundary layer ingested [7]. The size of engine's core was determined taking into account the systems that need to be allocated inside, following Jansen's study on sizing electric components for similar configurations [13], and the shape of the aft-fuselage. The fan was design as a simple stage compressor composed by 22 blades constructed through the methodology presented by Mattingly [14]. The rotation of the blades was set to 2000 rpm determined to avoid supersonic speeds at the tip of the blades considering a safety margin.

\section{Modelling}

The different elements of the aircraft configurations were modeled in Catia V5 and parametrized to enable rapid changes in size and geometry. The CAD models were imported into Ansys Workbench for meshing and simulations setup. The simulations were carried out on a HPC (High Performance Computer). 


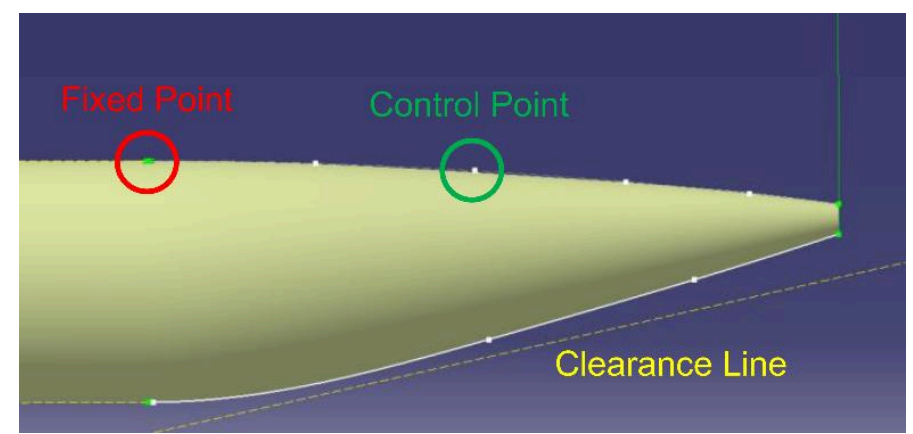

Fig 4. Fuselage CAD model parametrization

\begin{tabular}{llr}
\hline \hline \multicolumn{3}{c}{ Table 2: } \\
\hline \hline Parameter & Units & Value \\
\hline $\boldsymbol{\rho}_{\infty}$ & $\mathrm{kg} / \mathrm{m}^{3}$ & 0.32 \\
$\boldsymbol{U}_{\infty}$ & $\mathrm{m} / \mathrm{s}$ & 230.61 \\
$\boldsymbol{S}_{\text {ref }}$ & $\mathrm{m}^{2}$ & 137.85 \\
\hline
\end{tabular}

\subsection{Isolated Configuration}

The isolated configuration corresponds with the baseline airframe and the aft-fan such that both elements interact with separate flows and therefore, there is no aerodynamic interference between the aircraft and the electric propulsor. The modelling of the isolated configuration was performed through two different simulations divided in fuselage and electric fan such that both elements interact with different flows.

The CAD model of the fuselage was parametrized with a series of points to act as control points allowing the free deformation of the body, aiming to facilitate further optimization work (Fig 4). The boundaries of the deformation were constrained with several fixed points to maintain the volume required for the payload and the takeoff and landing clearance angle. The CAD model of the fuselage was then imported into Ansys Workbench, where a 3D unstructured mesh was generated composed of $25,367,151$ nodes and $18,783,158$ elements. The aim of the simulations of the fuselage was to determine the pressure distribution over the airframe as well as capturing the boundary layer profiles in longitudinal and radial directions. Therefore, the mesh was formed using the feature Inflation Layers on the fuselage's surfaces (Fig 5), which refines the mesh locally, creating thin layers near the walls to generate high aspect ratio cells, suitable for capturing areas where the airflow experiences rapid changes in critical variables such as within the boundary layer. Transient simulations were conducted to replicate flight conditions during cruise using the density solver included in the Ansys Workbench's module Fluent. The boundary conditions were specified as no-slip wall for the surfaces of the fuselage and pressurefar field for the surfaces of a control volume large enough such that the

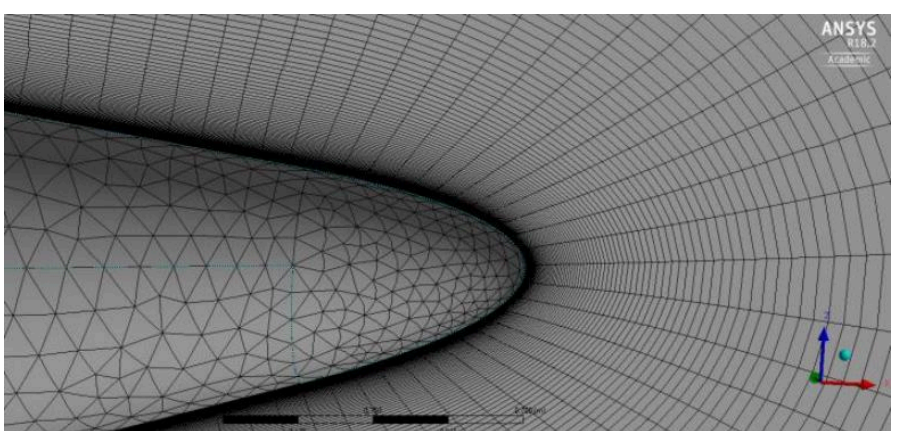

Fig 5. Rear fuselage mesh detail

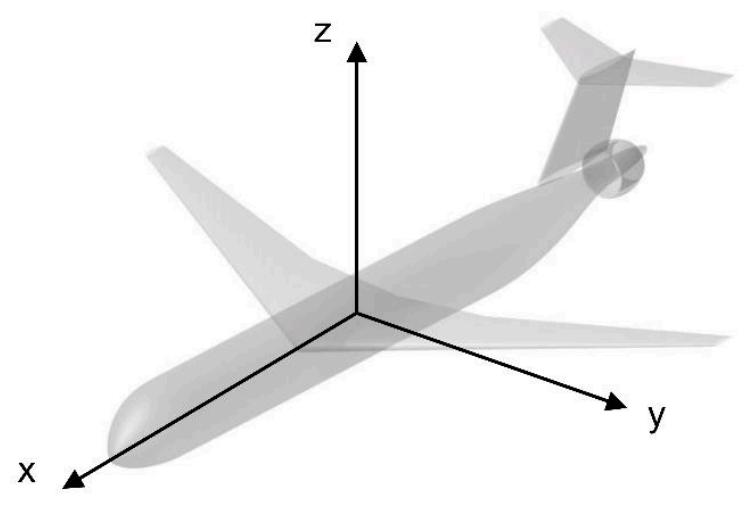

Fig 6. Reference axis system

properties of the flow correspond to the properties of the freestream, shown in Table 2.

A parametrized CAD model of the electric fan was also imported into Ansys Workbench. The CFD simulations were conducted using the sliding mesh technique, which allows the simulation of flows in multiple moving reference frames. To apply this technique, two different cell zones were created during the meshing phase, one to represent the static parts of the model and the other for the moving parts. The former included the far-field control volume, the nacelle and the engine core and the latest, included the fan blades and a cylindrical control volume around the fan blades. Both zones were bounded by all the faces of the fan control volume creating interface zones that limited both cell zones. The adjacent cells from different zones are related to each other by a mesh interface, allowing movement relative to each other. The mesh was composed of $17,427,518$ nodes and 12,562,991 elements.

\subsection{Integrated Configuration}

For the last set of simulations, the electric fan was integrated with the airframe. The fuselage shape was slightly modified to accommodate the fan at the tail cone. The direct coupling of the fan with tail cone produced sharp edges in the connection between the elements, therefore, it was necessary to adapt the shape of the rear fuselage to provide a smooth aerodynamic surface. Nevertheless, the modifications were minimum to ensure that the comparison with the isolated configuration was valid. The method followed to mesh the model was the same as the one 



Fig 7. Mach and pressure contours around fuselage (top), Mach contour rear fuselage detail (center) and Mach contours around electric fan (bottom) 

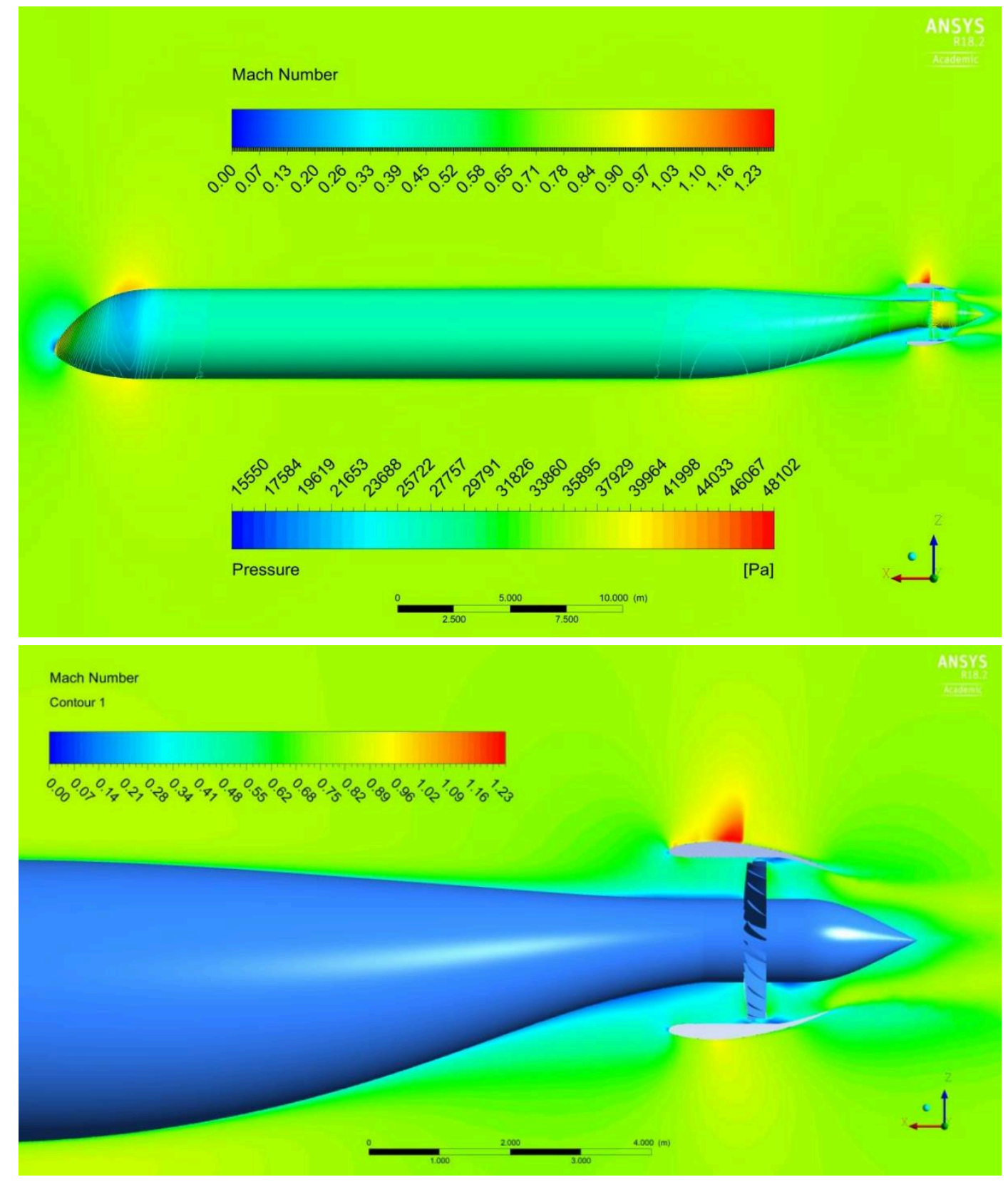

Fig 8. Mach and pressure contours around BLI configuration (top), Mach contours rear detail (bottom)

created for the fan and the fuselage to maintain consistence between the simulations. In this case, the mesh was composed of $31,787,908$ nodes and $18,783,158$ elements.

\section{Results and discussion}

From the simulation were extracted the forces integrated over the surfaces of the elements, the moments induced by the forces around the center of gravity and the total pressure at the fan-face. In addition, several contours were generated to better understand the data obtained.

The forces and moments were transformed to nondimensional coefficients using the reference values shown in Table 2. Fig 6 exhibits the reference systems selected. The convention of sign of the forces are positive as indicated in Fig 6 and the moments are considered to be positive when the rotation is produced in an anticlockwise direction.

\subsection{Aerodynamic coefficients}

The $X$ axis represents the direction of flight. Thus, a positive coefficient means that there is a propulsive force pushing the aircraft forward and a negative coefficient indicates a force in the opposite direction, drag.

The results of the simulations, shown in Fig 11, indicated that the BLI configuration offers an improvement in propulsive force. This improvement represents an increment in thrust of $33.15 \%$ in the BLI configuration relative to the isolated case. The improvement in the fuselage aerodynamics is even more remarkable, achieving a 
Pressure

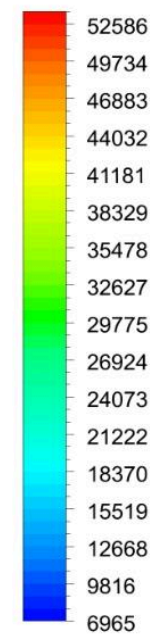

[Pa]

Pressure

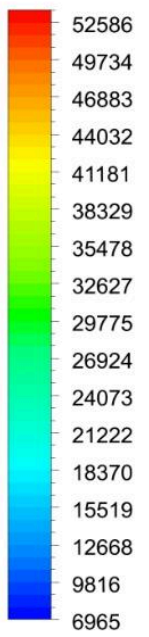

$[\mathrm{Pa}]$

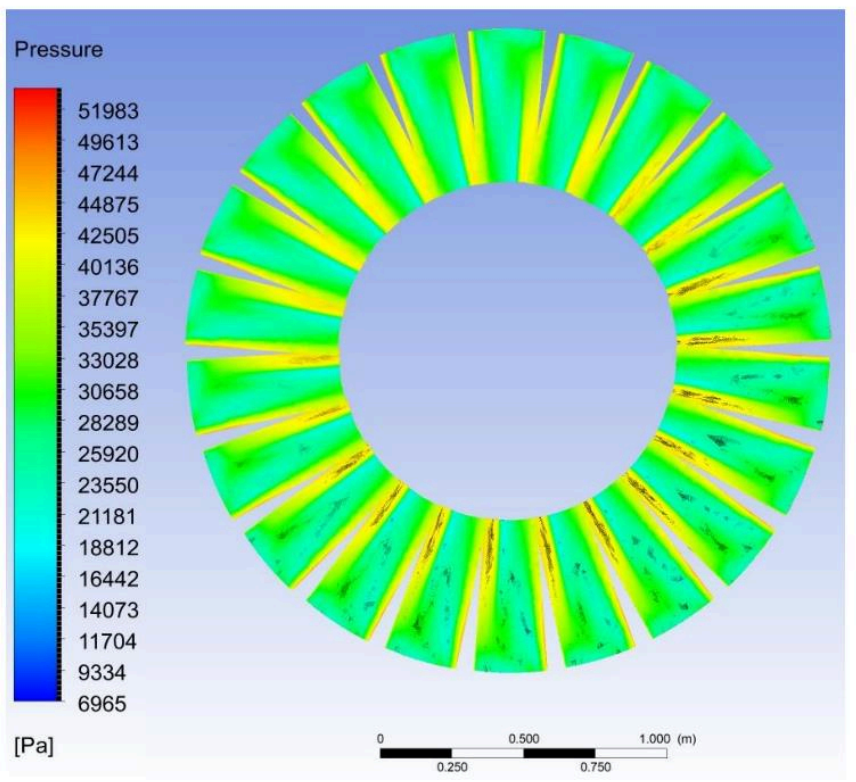

Fig 9. Pressure distribution over the nacelle (top) and fan blades (bottom) (Isolated configuration)
Pressure



15550

[Pa]

Pressure

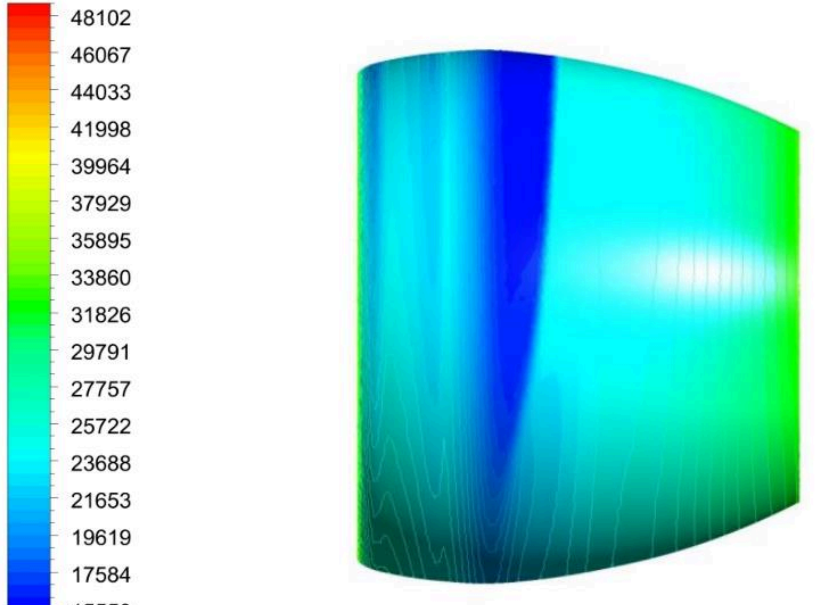

[Pa]

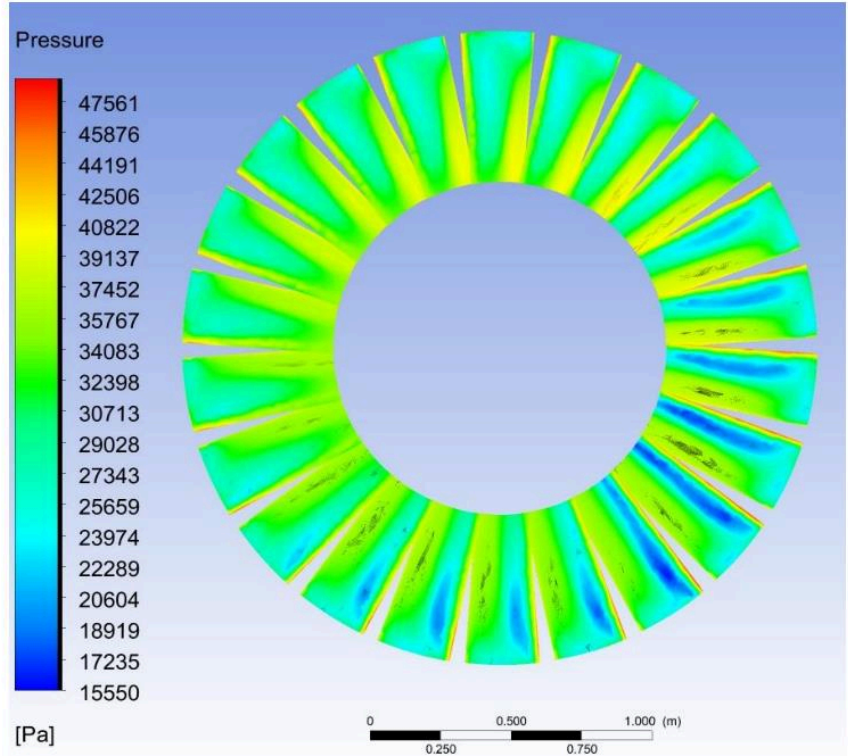

Fig 10. Pressure distribution over the nacelle (top) and fan blades (bottom) (Integrated configuration) 
Cx

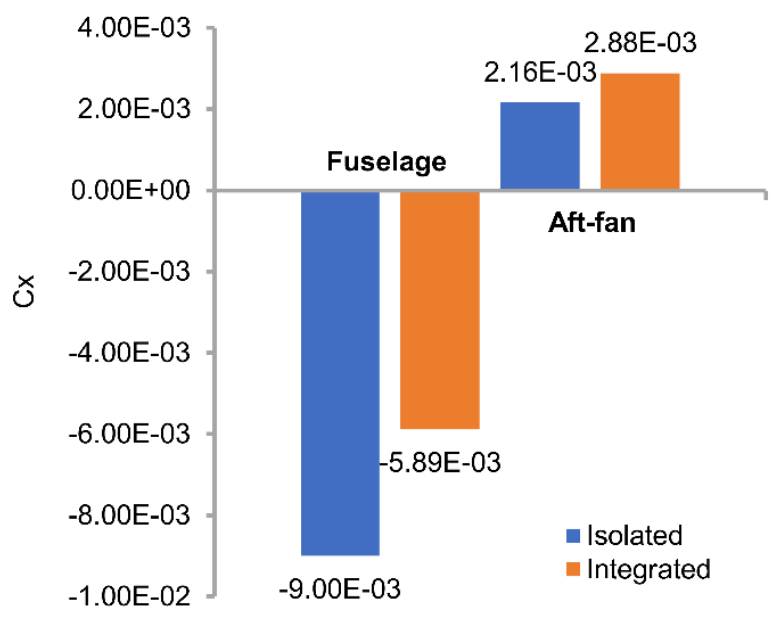

Fig 11. Aerodynamic coefficient in flight direction

$\mathrm{Cz}$

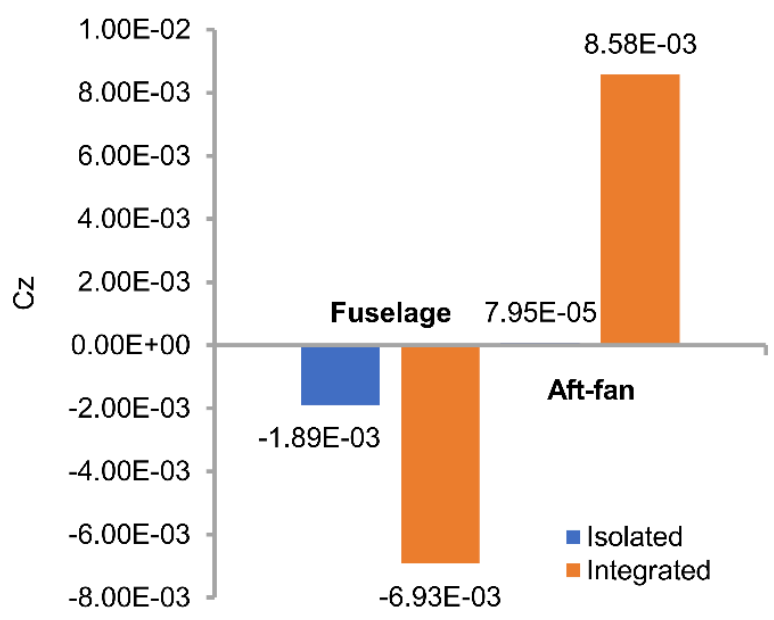

Fig 12. Aerodynamic coefficient in vertical direction

$34.58 \%$ of reduction in the force acting against the direction of flight, corresponding to the fuselage drag. The aerodynamic improvement, is due to a higher velocity in the aft section of the fuselage, which decreases the static pressure. In the case of the electric fan, the improvement is due to a reduction in the ram drag at the intake of the propulsor (Fig 7 and Fig 8). These results are in agreement with Gray's findings during his research on boundary layer ingestion with a similar configuration [8].

The changes in the lateral forces indicate an increment in both the fan and the fuselage (Fig 13). In the case of the fuselage the main reason is due to the presence of the aftfan, integrated with the airframe. The electric fan, not only increment in value but also changes in the direction.
Cy

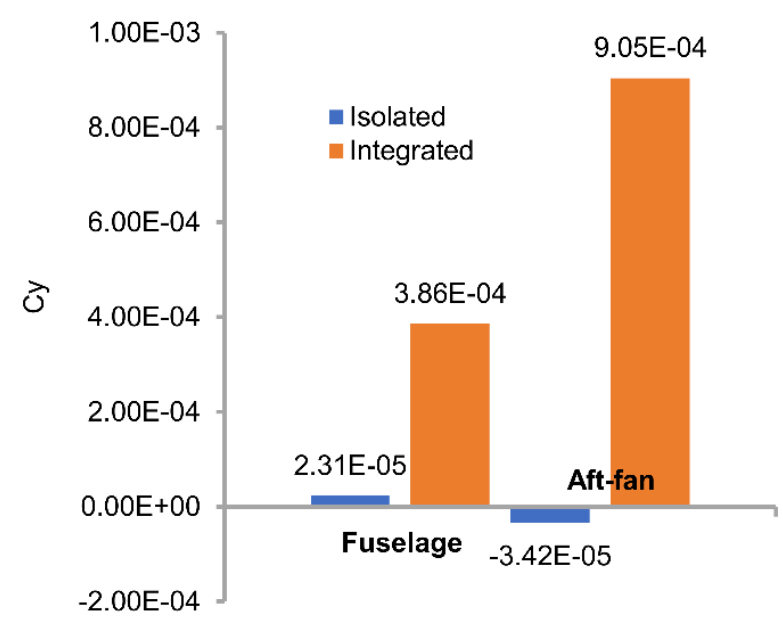

Fig 13. Aerodynamic coefficient in lateral direction

Fan-face total pressure

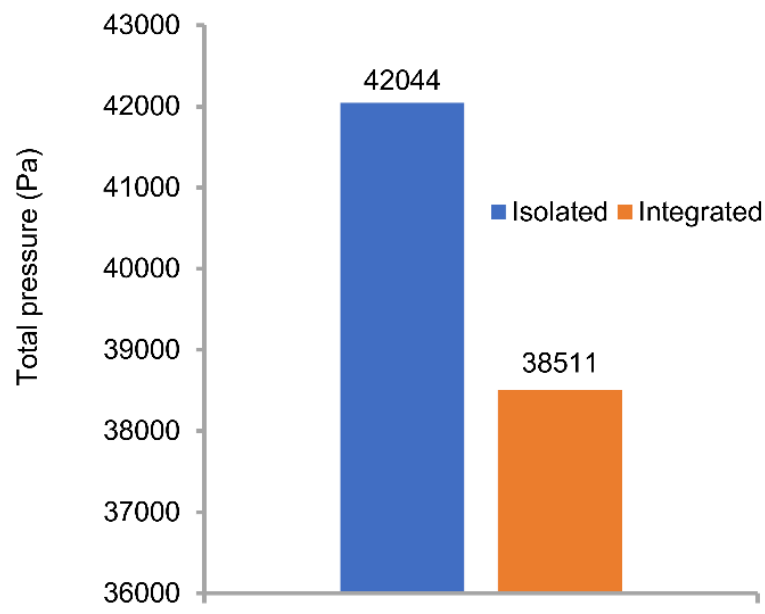

Fig 14. Total pressure at fan-face

Whereas in the isolated configuration there is a force acting on the negative direction of the $Y$ axis, the integrated configuration displayed a stronger force in the opposite direction (Fig 13). This change occurs because in the isolated configuration the main source of the force is the rotation of the blades and the pressure is distributed equally in each blade (Fig 9). The airflow entering the fan is clean and uniform in the isolated configuration but is highly turbulent when ingesting the boundary layer. Hence, in the integrated configuration the fan's blades are not affected by the same distribution of pressure as shown in (Fig 10), which creates a lateral force. In addition, the upswept geometry of the fuselage causes significantly different boundary layer profiles at different radial locations that may contribute to the generation of side forces (Fig 8). 
$\mathrm{Cmx}$

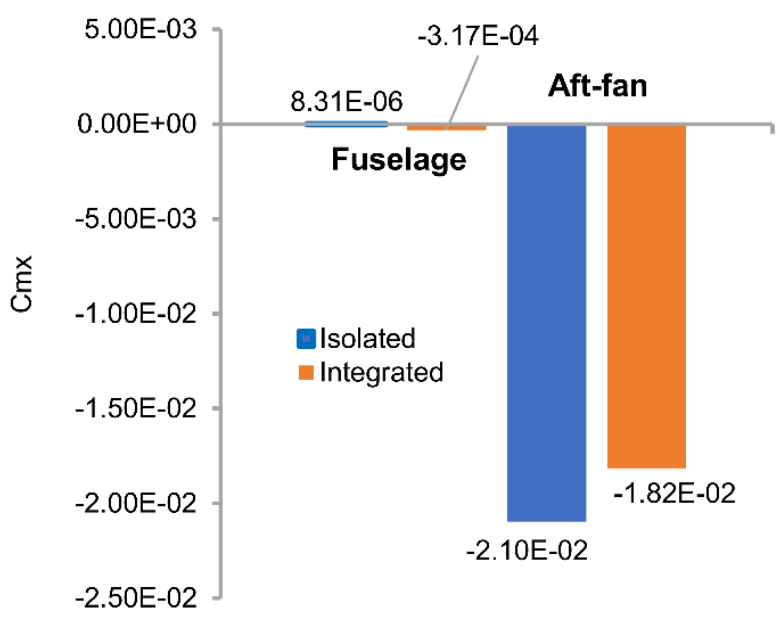

Fig 15. Rolling moment coefficient

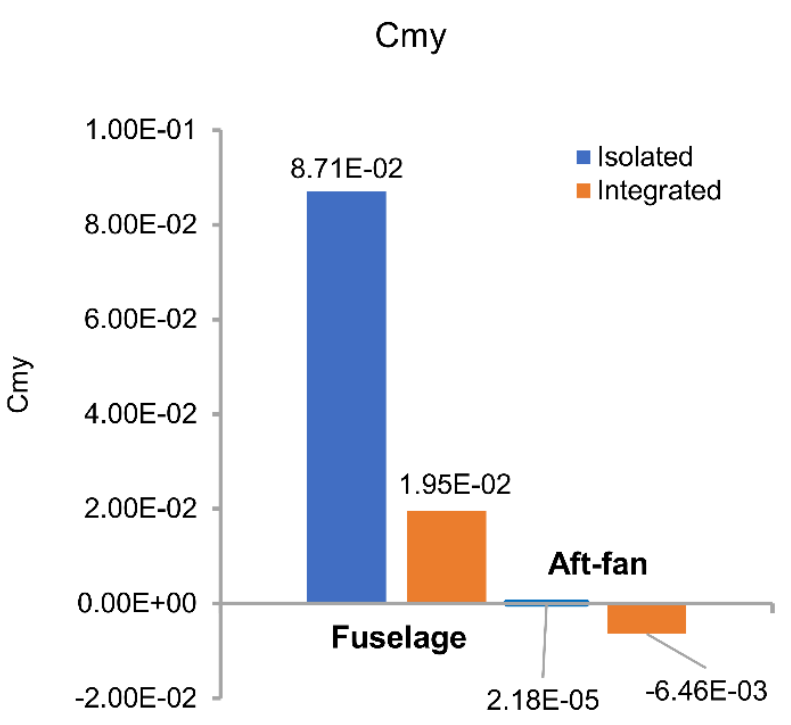

Fig 16. Pitching moment coefficient

The pressure distribution over the nacelle also presented an important variation between both configurations. In the BLI configuration the nacelle is immersed in the boundary layer, which, as it has been stated previously, is non-axisymmetric and the top surface of the nacelle is submerged in a faster airstream than the lower surface (Fig 8). This, generates a different pressure distribution in upper and lower surfaces of the nacelle of the fan, which induces a lifting force upwards that explains the changes in the vertical force for the propulsor. Similarly, the fuselage also experiments an increment in the vertical force of the integrated configuration with respect to the isolated configuration. The boundary layer is thicker in the lower surface than in the upper surface of the fuselage and therefore, the static pressure is higher (Fig 7), which push the airframe upwards.
$\mathrm{Cmz}$

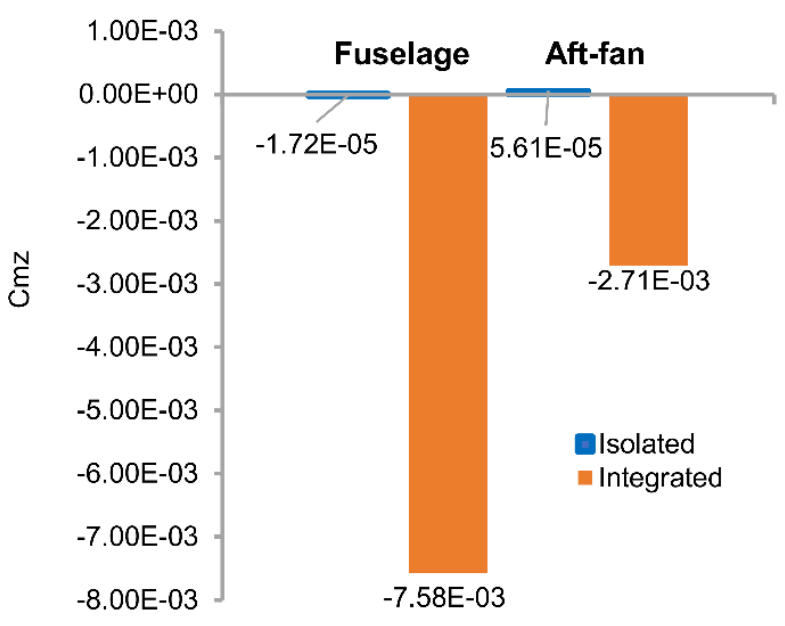

Fig 17. Yawing moment coefficient

This phenomenon is further enhanced in the integrated configuration since the presence of the electric fan thickens the boundary layer around the fuselage, especially in the lower surface (Fig 8).

\subsection{Moment coefficients}

The moments produced by the forces around the center of gravity of the fuselage and the engine were also recorded. during the simulations. The distribution of pressure and viscous forces varies greatly between both configurations. Not only does the highly distorted flow of the boundary layer around the rear of the fuselage induces a different distribution of forces over the engine but also the fuselage is affected by the coupling.

The results yielded an alleviation of the rolling moment produced on the electric fan in the BLI configuration relative to the isolated analysis. The rolling moment of the electric fan is mainly generated by the rotation of the blades and its relaxation is associated with a lower total pressure at the fan-face location while ingesting the boundary layer (Fig 14). The fuselage, is also affected by a variation in the rolling moment. During the isolated simulation, the rolling moment recorded was practically negligible. However, the rolling moment is enlarged by 2 orders of magnitude in the BLI configuration (Fig 15), this increment is due to the integration with the fan, which induces a rolling moment to the airframe.

There is also an increment in the pitching moment of the propulsor in the BLI configuration. However, after analyzing the forces, this boost was to be expected. The nacelle of the fan generates lift in the BLI model due to different velocity gradients at the top and the bottom surfaces (Fig 8), which induces a pitching moment in the aft-fan. On the other hand, the fuselage pitching moment is attenuated when the boundary layer is ingested by the propulsor (Fig 16). This 
effect is produced by the different distribution of pressure in both configurations.

Lastly, the yawing moment is largely increased for both the fuselage and the aft-fan in the BLI configuration (Fig 17). In the isolated configuration, the yawing moment is insignificant. In the case of the fuselage the yawing moment is probable induced by imperfections in the geometry and can be further reduced by an optimization process. The increment in yawing moment in the electric fan is consequence of the increasing lateral force generated by the distortion on the fan blades and the non-uniform distribution of pressure around the nacelle (Fig 10).

It may be that the results of the simulations are penalized by the non-optimized design and in future work, when more refined models are available, the trends may change or be alleviated. Nevertheless, the results highlight the importance of implementing 3D studies in boundary layer ingestion taking into account the integration of the airframepropulsor. The highly turbulent and non-axisymmetric flow found in the boundary layer at the rear of the fuselage precipitates changes in the behavior of the electric fan, changes that are further enhanced by the mere presence of the fan, which modifies the boundary layer profile, thickening it (Fig 8). The distribution of forces cannot be longer considered to be symmetric, and as a consequence, moments are generated. This needs to be accounted for in the design process due to the direct impact that the moments can potentially provoke in the stability and control of the aircraft.

\section{Conclusions}

The conceptual design of a narrow-body airliner with a boundary layer ingesting fan, located at the aft-fuselage, was developed for this work. The design was tested through CFD simulation to examine the benefits of this type of technology. First the elements were considered to be far away from each other such that there is no aerodynamic interference between the fuselage and the fan (isolated configuration). Then, CFD simulations were performed with the aft-fan integrated in the fuselage airframe. The aim of the isolated configuration was to act as a comparison point to evaluate the changes in aerodynamics and propulsion performance. The simulation consisted of 3D transient analyses using the sliding mesh technique to represent the rotation of the blades within an airflow.

The forces integrated over the surfaces of the aft-fan and the fuselage were obtained from the simulation as well as the moments generated by such forces around de center of gravities of the elements.

The boundary layer configuration results shown that there is a performance improvement in both the aerodynamics and the propulsion system. The performance improvement is $33.15 \%$ with respect to the isolated configuration. This increment in propulsive force is due to the slower airstream at the engine intake which reduces the ram drag. In addition, the nacelle is also surrounded by a slower flow, reducing the drag over it. In terms of aerodynamic improvement, the fuselage drag is reduced by $34.58 \%$ while ingesting the boundary layer around the fuselage. The aerodynamic improvement is due to the higher static pressure over the rear fuselage in the BLI configuration relative to the isolated analysis.

The results also yielded remarkable changes in the trends of the forces in the lateral and vertical direction as well as in the moments produced by these forces around the center of gravity of the fuselage and propulsor. The distribution of pressure around the fuselage and the velocity profile of the boundary layer varies largely from one configuration to the other. The presence of the propulsor at the aft-fuselage modifies the boundary layer, thickening it, and the upswept geometry of the aft fuselage creates a boundary layer with different profiles at each radial location, which generates a highly distorted flow entering the engine. In addition, the boundary layer is thicker on the lower surface than the top surface such that the lower surface of the nacelle is totally embedded in a slow-moving flow. On the other hand, the higher surface of the nacelle is immersed in a faster airstream. Thus, the distribution of pressure is nonaxisymmetric and contributes to the generation of forces in the lateral and vertical directions and the associated momentums. These effects, due to the non-axisymmetric shape of the fuselage, have not been captured before in any studies. Therefore, there was no possibility of comparing the results as in the case of the forces in the flight direction. It may be that in subsequence work, when more accurate and optimized designs are available, the impact of these forces and moments are less significant. However, this finding represents a first step in the impact of boundary layer ingestion on the stability and control of the aircraft.

The findings of this study highlight the importance of taking into account the integration of fuselage-propulsor to capture the variation of the boundary layer profile in presence of the aft-fan. Moreover, the results also displayed the relevance of analyzing the configuration assuming the threedimensional shape of the elements to capture the nonaxisymmetric properties of the flow, and therefore the nonaxisymmetric distribution of forces.

This work represents a first attempt to investigate the effects of ingesting the boundary layer around the fuselage beyond drag and propulsive force, introducing the analysis of all the forces and moments resulting from the integration airframepropulsor.

\section{References}

[1] J. Welstead, J.L. Felder, M.D. Guynn, W. Haller, M.T. Tong, S. Jones, I. Ordaz, J. Quinlan, B. Mason, Overview of the NASA STARC-ABL ( Rev . B ) Advanced Concept, One Boeing NASA Electr. Aircr. Work. (2017) 1-14. 
[2] C. Goldberg, D. Nalianda, D. MacManus, P. Pilidis, J. Felder, Method for simulating the performance of a boundary layer ingesting propulsion system at design and off-design, Aerosp. Sci. Technol. 78 (2018) 312-319. doi:10.1016/j.ast.2018.04.026.

[3] A. Abbas, J. De Vicente, E. Valero, Aerodynamic technologies to improve aircraft performance, Aerosp. Sci. Technol. 28 (2013) 100-132. doi:10.1016/j.ast.2012.10.008.

[4] A. Plas, D. Crichton, M. Sargeant, T. Hynes, E. Greitzer, C. Hall, V. Madani, Performance of a Boundary Layer Ingesting (BLI) Propulsion System, 45th AIAA Aerosp. Sci. Meet. Exhib. (2007). doi:10.2514/6.2007-450.

[5] M.K. Bradley, C.K. Droney, Subsonic Ultra Green Aircraft Research: Phase II. N+4 Advanced Concept Development, Mal. (2012) 207. doi:2060/20150017039.

[6] M.K. Bradley, C.K. Droney, Subsonic Ultra Green Aircraft Research: Phase II - Volume II - Hybrid Electric Design Exploration, Nasa/Cr-2015-218704. II (2011) 207. doi:2060/20150017039.

[7] J. Welstead, J.L. Felder, Conceptual Design of a Single-Aisle Turboelectric Commercial Transport with Fuselage Boundary Layer Ingestion, 54th AIAA Aerosp. Sci. Meet. (2016) 1-17. doi:10.2514/6.2016-1027.

[8] J.S. Gray, C.A. Mader, G.K. Kenway, J. Martins, Approach to Modeling Boundary Layer Ingestion using a Fully Coupled Propulsion-RANS Model, 58th AIAA/ASCE/AHS/ASC Struct. Struct. Dyn. Mater. Conf. (2017) 1-16. doi:10.2514/6.2017-1753.

[9] M. Drela, Power Balance in Aerodynamic Flows, AIAA J. 47 (2009) 1761-1771. doi:10.2514/1.42409.

[10] A. Arntz, O. Atinault, A. Merlen, Exergy-Based Formulation for Aircraft Aeropropulsive Performance Assessment: Theoretical Development, AIAA J. 53 (2015) 1627-1639. doi:10.2514/1.J053467.

[11] D. Howe, Aircraft conceptual design synthesis, Prof. Eng. (2000). doi:10.1002/9781118352700.

[12] D.P. Raymer, Aircraft Design: A Conceptual Approach, Fifth, American Institute of Aeronautics and Astronautics, Inc., Blacksburg, Virgina, 1992.

[13] R. Jansen, C. Bowman, A. Jankovsky, Sizing Power Components of an Electrically Driven Tail Cone Thruster and a Range Extender, 16th AIAA Aviat. Technol. Integr. Oper. Conf. (2016) 1-9. doi:10.2514/6.2016-3766.

[14] J.D. Mattingly, Aircraft Engine Design, 2002. http://books.google.com.tr/books?id=2Wy5rpdm3D MC. 
2020-02-21

\title{
Effect of a fuselage boundary layer
} ingesting propulsor on airframe forces and moments

\author{
Fernández, Antonio Martínez
}

Elsevier

Martinez Fernández A, Smith H. (2020) Effect of a fuselage boundary layer ingesting propulsor on airframe forces and moments. Aerospace Science and Technology, Volume 100, May 2020, Article number 105808

https://doi.org/10.1016/j.ast.2020.105808

Downloaded from Cranfield Library Services E-Repository 